\title{
NEW TECHNOLOGIES AND MECHANISMS FOR GREENHOUSES
}

\author{
Nikolay Bogatyrev, Sergej Morgun, Mikhail Poteshin, Dmitrij Semernin \\ Kuban State Agrarian University named after I.T. Trubilin, Russia \\ bogatyrevn@yandex.ru, morgun@bk.ru,mishadrive@mail.ru, y007ta@yandex.ru
}

\begin{abstract}
The number of greenhouses for the year-round production of vegetables, fruits, flowers is increasing in many countries worldwide. Currently, the technology of production in greenhouses is quite developed. Some difference in technology takes place due to the climatic conditions of the country and types of products produced. The cost of production depends on the technology used and energy costs. The purpose of the research is as follows: to determine the effectiveness of existing technologies, their impact on the cost of production, development of new technologies and mechanisms to reduce the cost of products and increase competitiveness. As a result of the research, it was established that the curtain mechanisms, as well as vent sash windows opening, fog and ventilation formation, consume not more than 7-9\% of electrical energy. The most part of electrical energy is used for artificial lighting system, soil moistening mechanisms, supplying of nutrient solution and for electric circulation pump of hot water in the heating system. According to the results of the research, an electric activator of aqueous solutions for disinfection and plant protection has been developed to increase the efficiency of growing products in greenhouses. The design of the system of mirrors for the equal distribution of the light flux from additional illumination throughout the entire volume of plants is substantiated. All technologies and mechanisms get power from a gas turbogenerator with an asynchronous generator. Main conclusions: the practical implementation of the developed technologies and mechanisms allows to reduce a consumption of electrical energy up to $17-19 \%$, depending on the geographical location of a greenhouse; the proposed development are new and it received 3 patents of the Russian Federation.
\end{abstract}

Keywords: greenhouse, technology, electrical energy.

\section{Introduction}

The daily quantity of photosynthetic active radiation (PAR) penetrating inside greenhouses for year-round production should be taken into consideration. There are many cases, when an additional artificial radiation shall be used. The type and capacity of artificial radiation systems should be chosen depending on a technology for vegetable and transplant seedlings production as well as in accordance with climate patterns of a construction district [1-13].

There should be minimal summed radiation (natural + artificial) not less than $25 \mathrm{~W} \cdot \mathrm{m}^{-2}$ PAR in vegetable transplant seedlings compartments of greenhouses. Daily quantity of PAR should be $250 \mathrm{~W} \cdot \mathrm{h} \cdot \mathrm{m}^{-2}$. The radiation level in vegetable greenhouses shall be not less than $70 \mathrm{~W} \cdot \mathrm{m}^{-2} \mathrm{PAR}$, daily quantity of PAR for green crop during the fruiting time is not less than $900 \mathrm{~W} \cdot \mathrm{h} \cdot \mathrm{m}^{-2} \mathrm{PAR}$. It is recommended to give radiation of $80 \mathrm{~W} \cdot \mathrm{m}^{-2}$ during the process of plants growing under the conditions of artificial radiation for plantlets and transplant seedling, as for green crop $-80-160 \mathrm{~W} \cdot \mathrm{m}^{-2}$ PAR [3;5].

Sustainable growth of plants in greenhouses and support of vital activity under conditions of changing environmental conditions are reached with a set of devices, which usually characterize stability of the development. Light conditions together with other environmental factors affect plant performance, as well as stability of its growth and development. The matter of light conditions effect can be checked with a content of chlorophyll in leaves of plants and their size. It is possible to cultivate plants effectively in the artificial environment only with using light technology with an additional source of light $[4 ; 7 ; 8]$.

The research [9;10] explains how different types of irradiators have an effect on the energy efficiency ratio coefficient in lettuce photoculture. The authors used irradiators with sodium-vapor lamp ДНа3-400-Супер and a LED irradiator as a light source with a percentage ratio of colors in the subrange: $30 \%$ - in blue; $20 \%$ - in green and $50 \%$ - in red. The level of effectiveness from using sodium-vapor lamp is $32.4 \%$ higher in comparison with using LED irradiator according to high yields of lettuce.

LEDs with unspecified ranges has found wide application in photoculture as sources of radiation. The cost of irradiation devices based on LEDs is quite expensive. A cheaper source is luminescent lamp, however, there is no radiation with a proper for plants wave length in its range. They use combined irradiators with different source types for solving the problem [6;10-12]. 
There are devices with sources located on leaves upper layer and a side supplementary lighting with additional artificial light sources to support physiological activity of leaves middle and lower layers and to enhance the effectiveness [12-16].

There are different types of pesticide used to protect plants in greenhouses. The main method of spreading different types of pesticide is spraying. The effectiveness of spraying depends on the amount, quantity and spreading drops of liquid on a surface. The most popular way of spraying is the fine-droplet spraying (droplet diameter 51-151 $\mu \mathrm{m}$ ). It is recommended to use activated water to reach such parameters; electrons in activated water are overactive, also, activated water has evident properties of reducing agent [17-19].

Due to researches, it is established that the value of $\mathrm{pH}$ index affects differently on the speed of disintegration rate of pesticide. Some herbicides disintegrate faster in alkaline medium and some of them - in acid medium. According to recent researches, the effectiveness of some herbicides and pest control chemicals reaches a higher level, if they dissolve in water with $\mathrm{pH}$ equal to 4 units. For example, glyphosate herbicide is more effective with $\mathrm{pH}$ 6-7. Each product has its specific characteristics of efficiency and there is $\mathrm{pH}$ index among them. Activated water has a high dissolving ability of action spectrum due to high (heavy) electric charges [17-19].

\section{Results and discussion}

We developed a device for inter-lighting of greenhouse plants with the help of local lighting of plants on the background of general lighting (fig. 1) [20].

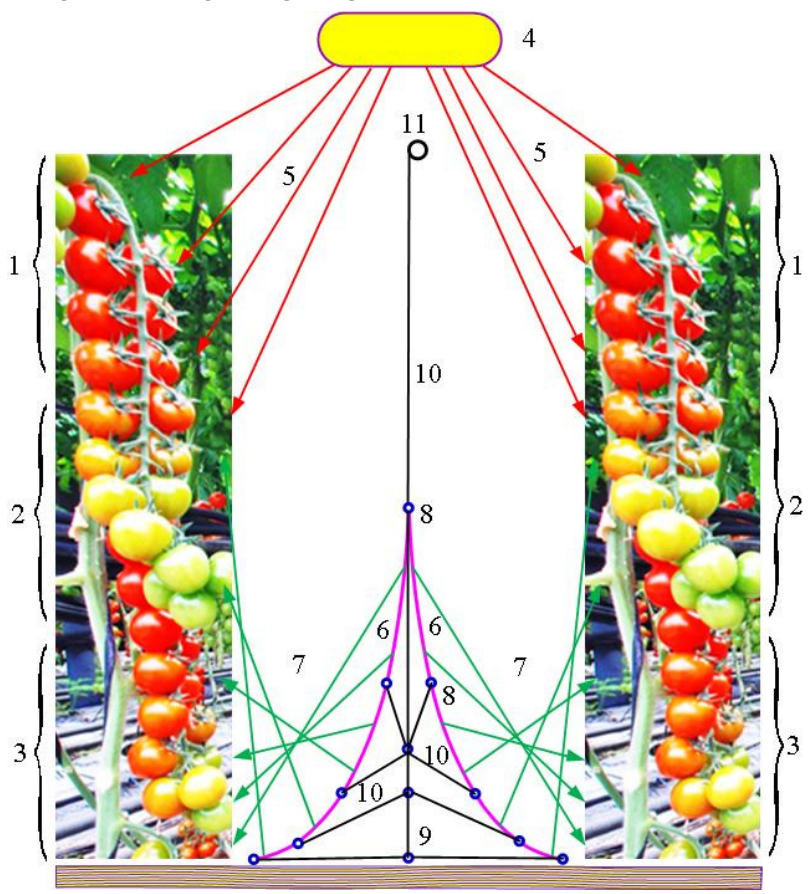

Fig. 1. The device for inter-lighting of greenhouse plants: 1 - upper layers of plants; 2 - middle layers of plants; 3 - lower layers of plants; 4 - a source of artificial light; 5 -direct rays of a source of light; 6 - two mirrors with changing curvature; 7 - reflected rays from inter-lighting; 8 - rotary joints;

9 - a mirror controlling device; 10 - moving length-adjustable bars; 11 - a mirror riser

There is one of high-pressure sodium-vapor lamps, reflector lamp ДНа3 or lamps with LEDs of high capacity used as a source of artificial light 4 . The mirror controlling device 9,6 includes moving length-adjustable bars 10 , which are fixed between rotary joints 8 . The mirror riser 11 is produced as a reversible gear motor (motor-reducer). Two mirrors with changing curvature 6 are made of materials with high light reflection coefficient, and, with the help of rotary joints 8 , the mirror controlling device 9 changes an angle from 10 (up) to 80 (down) degrees relative to the vertical [20].

There should be used large amount of electrical energy to make water electrically initiated [1719]. There is a electrolytic process of aqueous salt solutions device, which was developed to increase efficiency (Fig. 2,3) [21]. 


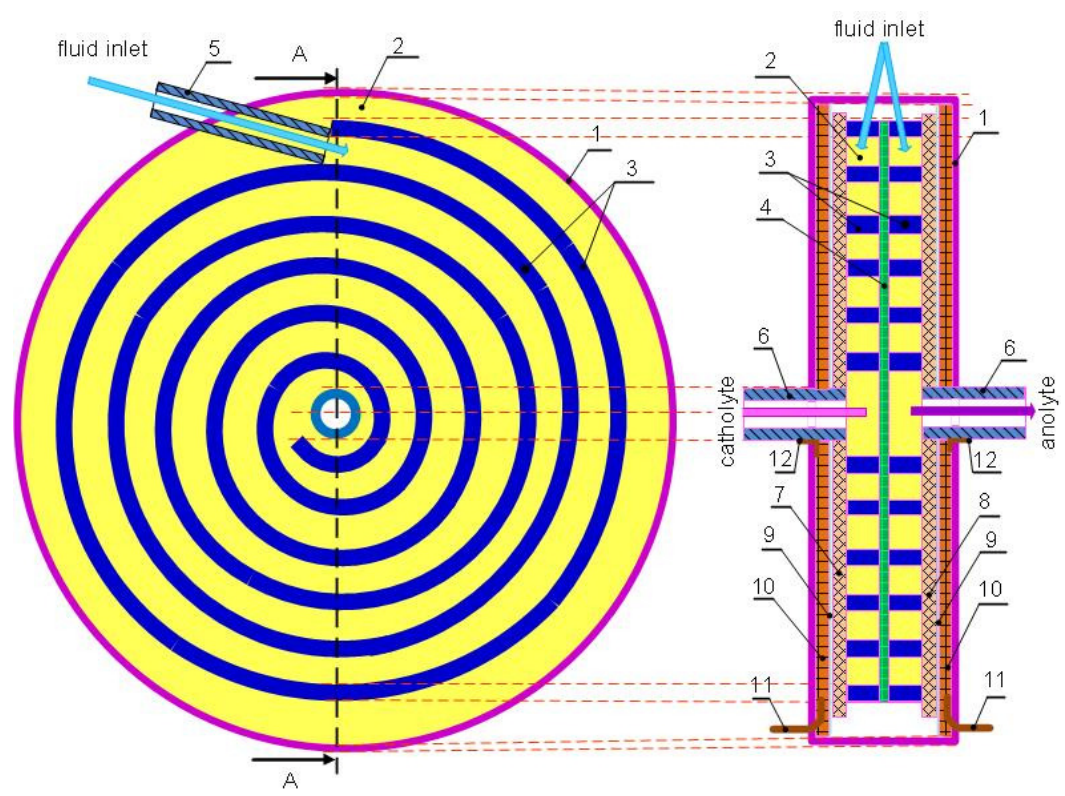

Fig. 2. The electrolytic process of aqueous salt solutions device: at the left - sectional view of the device; at the right - sectional view of the device A-A.

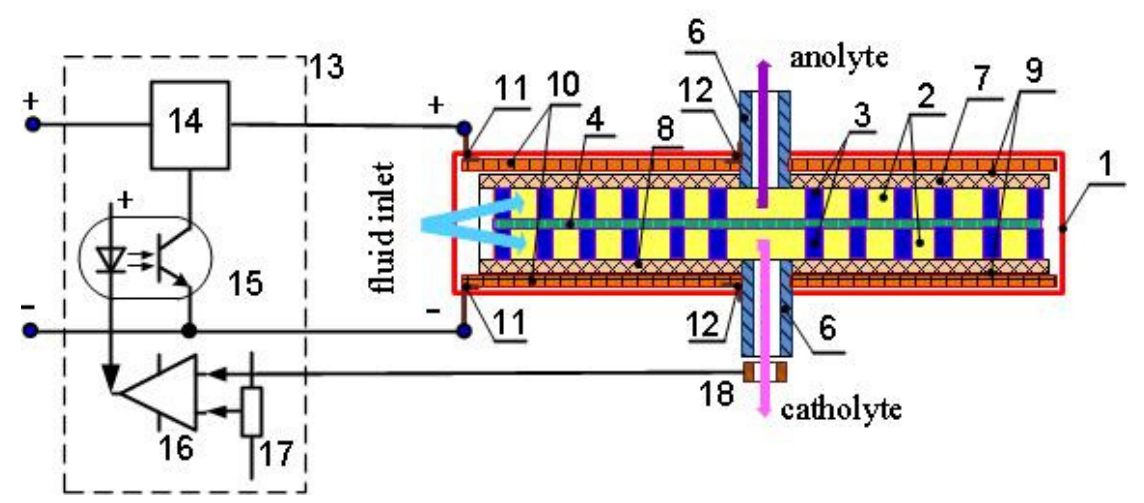

Fig. 3. Functional diagram of device operation

The electrolytic process of aqueous salt solutions device has flat cylindrical housing 1, membraneous electrochemical reactor 2 is made as two flat wrap springs 3 , which are separated with rounded fine-porous membrane 4, which has inlet 5 and outlet fittings (nozzles) 6, rounded anolyte 7 catholyte 8 , gaskets 9 are used to press flat or lateral (bank) concentric windings to electrodes 10 with outlets 11 and 12, the $\mathrm{pH}$ controlling system 13, which includes a controlled power source 14, photoncoupled pair (PCP) 15, an amplifier 16, a potentiometer 17, a conductivity probe 18 [21].

Flat concentric windings 10 with outlets 11 for connecting to the power source, and outlets 12 for connecting to the reactor with metal outlet fittings 6 . They are made of winding wire (bus), section of which is defined with reactor current 2. To make their structure more hard they are cast with compound and sintered. One winding is wrapped in a clockwise direction, the second one - counter clockwise. It is made so that with a serial connection, according to a construction, their magnetic fields would be summarized. See [21] for a detailed information.

Advantages of the device.

- Liquid (fluid) runs through annular channel, it is cycling there. There is a vortex motion under the action of centrifugal forces, which provides a better contact with electrodes.

- Ion transfer process in moving solution differs from neutral dissolved particles transfer process. Electrical and magnetic field have impact on motion of ion. Thus, ion transfer in the solution is made with convection, diffusion of ions to electrode in electrical and magnetic fields. 
- Flat concentric windings have small active resistance in comparison with water solution. That is why the losses are negligible, however, due to created electromagnetic field, the electrolysis process is increased sharply and efficiency coefficient of the device is increased as well.

Nowadays some of greenhouse complexes use gas reciprocating power plants with synchronous generators for matters of heating and electric power supply. Advantages of such decisions is obvious.

We offer to use asynchronous generators (AG) instead of synchronous generators in gas reciprocating power plants, asynchronous generators (AG) should be based on energy-efficient asynchronous engines with a square-cage rotor and condensing feeding. The main weak point of asynchronous generators is a steeply-deepening external characteristics [22-24], which is solved in our patent [25] (Fig. 4). operation of the device is in [25].
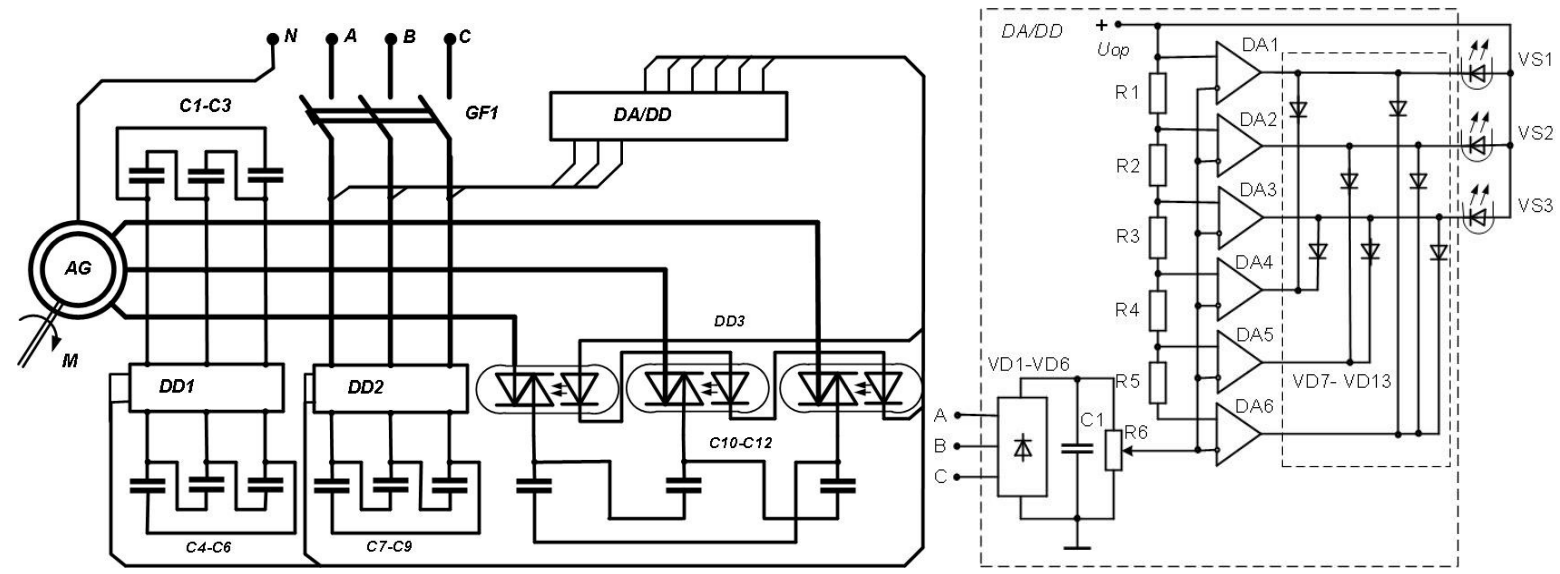

Fig. 4. Functional diagram of the device for regulation and stabilization of voltage of a selfsupporting asynchronous generator: $A G$ - asynchronous generator; $D D 1-D D 3$ - three-phase solid relay (switch) with voltage phasing control via "zero"; $G F 1$ - automatic circuit-breaker; $D A / D D$ control unit; $C 1-C 3$ - feeding condensers; $C 4-C 12$ - switching capacitors; VD1-VD6 - reverse circuit

diodes; DA1-DA6 - comparators; $R 1-R 5, R 6$ - controlled standard-voltage divider; $C 1$ - filter capacitor; VD7-VD13 - decoding device; VS1-VS3 - optic couplers of solid state relays DD1-DD3

Calculation example for asynchronous motor (AG) on base of motor series $R A 355 M L B 4$ with the capacity of $400 \mathrm{~kW}$; capacity coefficient 0.88 ; efficiency coefficient $=96.4$; mass $=2015 \mathrm{~kg}$.

Capacity of condensers, providing self-feeding of AG no-load (at idle) and reactive load power compensation are calculated according to the formula:

$$
C_{\Sigma}=\frac{P_{N}\left(\operatorname{tg} \varphi_{A G}+\operatorname{tg} \varphi_{H}\right)}{2 \pi f m U_{c}^{2}} 10^{6}, \mu \mathrm{F}
$$

where $P_{N}$ - capacity from a generator, $\mathrm{W}$;

$U_{c}$ - voltage of a condenser, $\mathrm{V}$;

$f$ - current frequency, $\mathrm{Hz}$;

$m$ - number of phases, $\cos \varphi_{A G}=0.88,\left(\varphi_{A G}=31.5^{\circ}\right)$ and $\cos \varphi_{N}=0.8,\left(\varphi_{A G}=41^{\circ}\right)-$ generator capacity coefficient and loading factor.

For rated load:

$$
C_{\Sigma}=\frac{400000\left(\operatorname{tg} 31.5^{\circ}+\operatorname{tg} 41^{\circ}\right)}{23.14503380^{2}} 10^{6}=3796 \mu \mathrm{F}
$$

Capacity of condensers, providing self-feeding of AG no-load (at idle):

$$
\begin{gathered}
C_{V}=\frac{P_{N} \operatorname{tg} \varphi_{A G} 10^{6}}{2 \pi f m U_{c}^{2}}, \\
C_{V}=\frac{4000000,5410^{6}}{23.14503380^{2}}=1588 \mu \mathrm{F}
\end{gathered}
$$


An additional voltage-controlled capacitance is defined as a difference in total capacity for operation with rated load capacity and capacity of feeding.

$$
\begin{gathered}
C_{D}=C_{\Sigma}-C_{V} . \\
C_{D}=3796-1588=2208 \mu \mathrm{F} .
\end{gathered}
$$

In accordance with a diagram of AG power circuit, an additional capacity of bank of capacitors shall be divided on three parts:

$$
C_{D 1}=368 \mu F, C_{D 2}=736 \mu F, C_{D 3}=1104 \mu F,
$$

While control unit works, capacity is switched according to algorithm:

$$
368 \Rightarrow 736 \Rightarrow 1104 \Rightarrow 1472 \Rightarrow 1840 \Rightarrow 2208 \mu F \text {. }
$$

AG external characteristics with capacity of $400 \mathrm{~kW}$ are shown on Fig. 5.

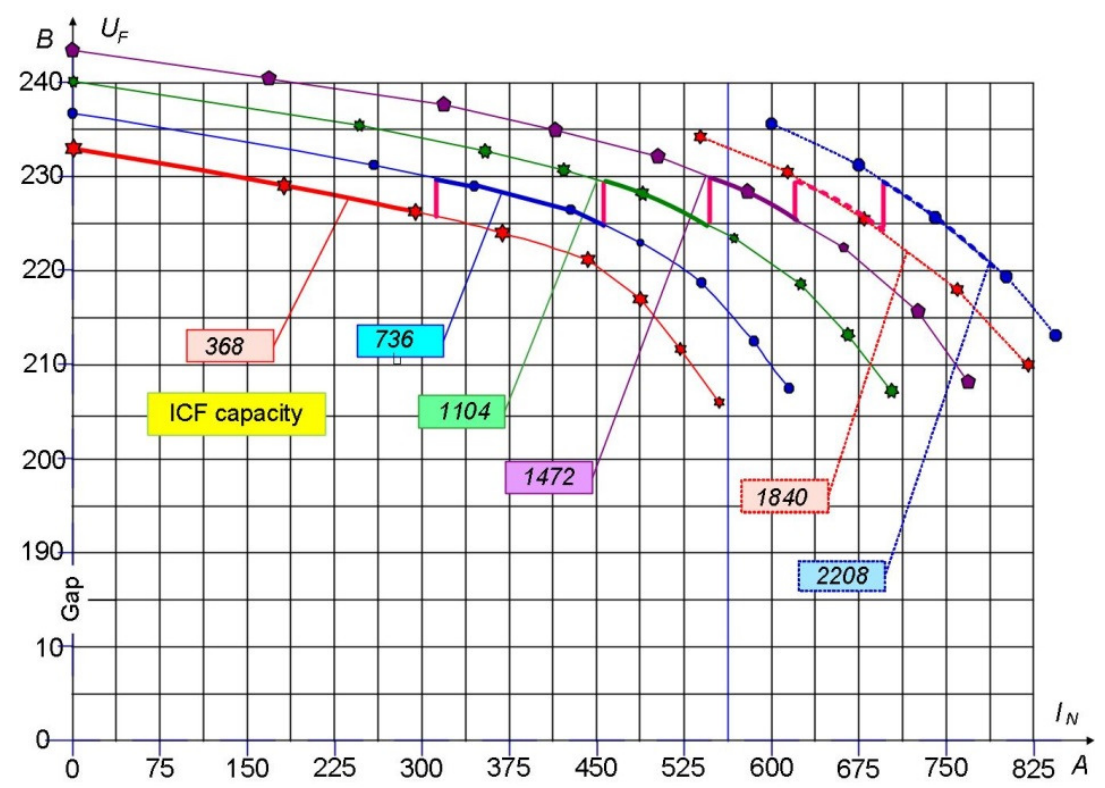

Fig. 5. AG external characteristics with capacity of $400 \mathrm{~kW}$

\section{Conclusions}

Introduction of innovative researches and projects for year-round production greenhouses will help to reduce productive costs and to lower production costs.

1. Devices for inter-lighting of greenhouse plants without additional sources of electrical energy allows to increase the efficiency of using artificial lighting to light when cultivating plants. The reflected rays of light from the mirrors support the physiological activity of the leaves of the middle and lower tiers of plants, providing intensive formation of fruits.

2. A new construction of the device for the electrolysis of aqueous salt solutions reduces the specific energy consumption from $q=5.7 \cdot 10^{-3}$ to $2.9 \cdot 10^{-3} \mathrm{~kW} \cdot \mathrm{h} \cdot \mathrm{l}^{-1}$ with the same performance and hydrogen indicator.

3. Devices for regulating and stabilizing the voltage of an autonomous asynchronous generator applicable to generators with a capacity of from units to hundreds of kilowatts. The advantage is that the switching of capacitors occurs when the voltage passes through zero. Therefore, there are no harmonic components in the output voltage.

\section{References}

[1] Guidelines for technological design of greenhouses and greenhouse plants for growing vegetables and seedlings. RD-APK 1.10.09.01-14, Moscow 2014 - 104 p. (In Russian) 
[2] Rakutko S., Alsina I., Avotins A., Berzina K. Manifestation of effect of fluctuating asymmetry of bilateral traits of tomato growing in industrial greenhouses / Engineering for rural development jelgava, 23.-25.05.2018 pp. 186-191.

[3] Mishanov A.P., Markova A.E. The influence of various types of feeds on the coefficient of energy-ecological compatibility in light-growing lettuce. Technologies and technical means of mechanized production of crop and livestock products. 2017. No. 92. pp. 25-31. (In Russian)

[4] Alves-Silva E. The influence of Ditylenchus (Nematoda) on the fluctuating asymmetry of Miconia fallax (Melastomataceae). Ecología Austral, vol. 22, 2012, pp. 53-61.

[5] Rakutko S.A., Mishanov A.P., Rakutko E.N., Markova A.E. Justification of the parameters of the combined phytoradiator / Technologies and technical means of mechanized production of crop and livestock. 2017. No. 92. P. 46-56.

[6] Stepanchuk G.V., Yudaev I.V., Zharkov A.V. Energy Efficient Irradiation System in a Greenhouse // Don Agrarian Science Bulletin "Technologies, Means of Mechanization and Power Equipment”-2016 - № 1 (33), p. 5-12.

[7] H.G. Choi, B.Y. Moon, N.J. Kang Effects of LED light on the production of strawberry during cultivation in a plastic greenhouse and in a growth chamber Sci. Hortic., 189 (2015), pp. 22-31.

[8] Venâncio H., Santos J.C., Alves-Silva E. Leaf phenotypic variation and developmental instability in relation to different light regimes. Acta Botanica Brasilica. 2016. Volume 30. Number 2. pp. 296-303.

[9] Moura R.F., Alves-Silva E., Del-Claro K. Patterns of growth, development and herbivory of palicourea rigida are affected more by sun/shade conditions than by cerrado phytophysiognomy. Acta Botanica Brasilica. 2017. . Volume 31, Number 2, pp. 286-294.

[10] Andrei A.Ye., Leonid B.P., Ivan G.T. Spectral aspect when using light-emitting diode irradiators for salad plant cultivation under photoculture conditions. Light \& Engineering. 2015. Volume 23. Number 4. pp. 55-62.

[11] Prikupets L.B., Boos G.V., Terekhov V.G., Tarakanov I.G. And biochemical composition of green salad foliage biomass. Light \& Engineering. 2018. Volume 26. Number 4. pp. 38-47.

[12] Dolgikh P.P., Samoilov M.V. Expansion of the functions of irradiation facilities when using greenhouses in the microclimate control system / Vestnik IrSAA. - 2016. No. 72, p. 130-138.

[13] Sudakov V.L., Anikina L.M., Udalova OR, Shibanov DV Optimization of the light environment when growing plants in terms of phytotrons / Gavrish 2012 - No. 3, p. 14-16.

[14] Patent RU 2318368 IPC A01G 9/24 Device for irradiating plants / Long PP, Shilonosova T.Yu., Pilchuk N.A. // Published: 10.03.2008 Bull. № 7. - 6 p.

[15] Patent RU 2328652 IPC F21V 21/02 The device leveling the degree of exposure in industrial premises / Stepanchuk GV, Klyuchka EP, Yakusheva E.E. // Published: 07/10/2008 Byul. № 19 $6 \mathrm{~s}$.

[16] Patent RU 2490868 IPC A01G 7/04 A way to increase the productivity and profitability of growing cucumber in green conditions in the north / E. Grigoray, E. Butkin, V. Dalke, I. V. // Published: 08.27.2013 Bull . № 24. - 9 p.

[17] Oskin S.V. Development of a mathematical model of an electroactivator taking into account data on water quality and requirements for a working solution of a herbicide / S.V. Oskin, N.Yu. Kurchenko // Polythematic network electronic scientific journal of the Kuban State Agrarian University [Electronic resource]. - Krasnodar: KubSAU, 2015. - №03 (107). - IDA [article ID]: 1071503079. - Access mode: http://ej.kubagro.ru/2015/03/pdf/79.pdf.

[18] Oskin S.V. Engineering methodology for determining the parameters of an electroactivator / S.V. Oskin, N.Yu. Kurchenko // Polythematic network electronic scientific journal of the Kuban State Agrarian University [Electronic resource] .- Krasnodar: Kuban State Agrarian University, 2015. — No. 03 (107) .- IDA [article ID]: 1071503080.- Access mode: http: // ej. kubagro.ru/2015/03/pdf/80.pdf.

[19] Patent RU 2475456 IPC C02F 1/461 Installation for the electrolysis of water-salt solutions / Normov DA, Kurzin N.N., Shuskin E.I., Oskin A.S. Posted 02/20/2013; Bul. No. 50. - 5 s.

[20] Patent RU 2629755, IPC A01G 7/04, A01G 9/20, F21V 14/02 Device for inter-row lighting of greenhouse plants / Bogatyrev N.I., Gish R.A., Morgun S.M., Semernin D. Yu, Potapenko Yu.V., Chumak M.S. Posted 09/01/2017; Bul. No. 25. - 9 c. 
[21] Patent RU 2660440 IPC C25B 1/10, C25B 11/02, C25B 9/08 A device for the electrolysis of water-salt solutions / Bogatyrev N. I., Oskin S. V., Tarasenko B. F., Tsokur D. S., Blyagoz A. A., Dovchenko D. V. Published 06/07/2018 Bull. № 19. - 9 p.

[22] Ambarnath Banerji, Sujit K. Biswas, and Bhim Singh Voltage and Frequency Controller for An Autonomous Asynchronous Generator / International Journal on Electrical Engineering and Informatics - Volume 6, Number 2, June 2014, pp. 250-265.

[23] Sonam Singh, A.N. Tiwari Voltage and frequency controller for self excited induction generator in micro hydro power plant: review International Journal of Advanced Research in Electronics and Communication Engineering (IJARECE) Vol. 2, Issue 2, February 2013, pp. 214-219.

[24] Vitaly Spitsa, Alon Kuperman, George Weiss and Raul Rabinovici Design of a Robust Voltage Controller for an Induction Generator in an Autonomous Power System Using a Genetic Algorithm. Proceedings of the 2006 American Control Conference Minneapolis, Minnesota, USA, June 14-16, 2006, pp. 3476-3481.

[25] Patent RU 2373630, IPC H02P 9/46, H02J 3/18 A device for regulating and stabilizing the voltage of an autonomous asynchronous generator / Bogatyrev, NI, Barakin, NS, Vronsky, AV, Grigorash, A.O. , Poteshin M.I. Posted 11/20/09; Bul. No. 32. - 7 c. 